\title{
Entrainment Probabilities of Mixed-Size Sediment Incorporating Near-Bed Coherent Flow Structures
}

\author{
Fu-Chun $\mathrm{Wu}^{1}$ and Kuo-Hsin Yang ${ }^{2}$
}

\begin{abstract}
In this work we incorporate the effect of near-bed coherent flow structures into the entrainment of randomly configured mixed-size sediments. The fourth-order Gram-Charlier type probability density function (GC pdf) of near-bed streamwise velocity is employed to account for the higher-order correlations associated with turbulent bursting. A compilation of the published data over a wide range of bed roughness is used to analyze the near-bed coherent flow structures, including the second-, third-, and fourth-order moments of velocity fluctuation (i.e., turbulence intensity, skewness, and flatness factors) required in the fourth-order GC pdf. An important result of this study is a set of quantitative relations used to predict these higher-order moments as a function of the roughness Reynolds number. The random grain protrusion is parameterized with the exposure and friction heights, and an existing probabilistic approach is used to correct the hiding effect of mixed-size sediment. The above factors are all incorporated into the formulation of entrainment (rolling and lifting) probabilities. As compared to the previous normal and lognormal models, the present results demonstrate significantly improved agreement with the observed data for the unisize and mixed-size sediments under partial- and full-transport conditions. The results also reveal that the third-order GC pdf can be used to approximate the fourth-order one for the fully rough beds, however, for smooth beds the fourth-order GC pdf should be used to adequately incorporate the effects of higher-order correlations. This paper offers some new insights into the processes of sediment entrainment.
\end{abstract}

DOI: 10.1061/(ASCE)0733-9429(2004)130:12(1187)

CE Database subject headings: Entrainment; Sediment; Turbulence; Bed roughness; Correlation.

\section{Introduction}

The initiation of sediment motion has long been an attractive topic for hydraulic researchers involved in the prediction of sediment transport. The probability of sediment entrainment is among the most important components of the stochastic bedload models (e.g., Einstein 1950; Paintal 1971; Sun and Donahue 2000; Wu and Yang 2004). In recent years, a series of work addressing the entrainment probabilities of unisize sediments have been presented (e.g., Cheng and Chiew 1998; Papanicolaou et al. 2002; Wu and Lin 2002; Wu and Chou 2003a). In their theoretical formulations, different forms of probability distribution for the fluctuating turbulent velocities were used, or various bed-packing conditions (grain configurations) were considered. Through these studies, substantial improvements in the evaluation of entrainment probabilities have been made, and some insights into the incipient motion are acquired.

However, to date two key factors affecting the incipient motion of natural sediments still remain to be incorporated into the

${ }^{1}$ Associate Professor, Dept. of Bioenvironmental Systems Engineering and Hydrotech Research Institute, National Taiwan Univ., Taipei 106, Taiwan, R.O.C. E-mail: fcwu@ntu.edu.tw

${ }^{2}$ Research Assistant, Dept. of Bioenvironmental Systems Engineering, National Taiwan Univ., Taipei 106, Taiwan, R.O.C.

Note. Discussion open until May 1, 2005. Separate discussions must be submitted for individual papers. To extend the closing date by one month, a written request must be filed with the ASCE Managing Editor. The manuscript for this paper was submitted for review and possible publication on November 6, 2003; approved on July 28, 2004. This paper is part of the Journal of Hydraulic Engineering, Vol. 130, No. 12, December 1, 2004. CASCE, ISSN 0733-9429/2004/12-1187-1197/ $\$ 18.00$. theoretical formulation of entrainment probabilities, which include the effects associated with near-bed coherent flow structures and randomly configured mixed-size sediments (e.g., Ling 1995; Cheng and Chiew 1999; Wu and Chou 2003a). The former is typically characterized by the periodic bursting events such as sweeps, ejections, and inward/outward interactions (e.g., Nelson et al. 1995; Papanicolaou et al. 2001); the latter is represented by the random grain protrusion, friction angle, and hiding-exposure effect (Kirchner et al. 1990; Wu et al. 2000). The advances in the experimental, computational, and theoretical works made in the last three decades as well as some latest findings now provide us chances to tackle such challenging problems.

In this study we incorporate the effect of near-bed coherent structures into the entrainment probabilities of mixed-size sediments. A compilation of experimental and simulation data for a wide range of bed roughness are used to analyze the higher-order correlations associated with turbulent bursting. The random configuration of mixed-size sediment is parameterized using the existing probabilistic approaches. The model results are then compared with the published data for unisize and mixed-size sediments under various transport conditions.

\section{Near-Bed Coherent Flow Structures}

A turbulent flow is typically characterized by its intensity, i.e., the root-mean-square value of velocity fluctuations. If the turbulent velocities were independently random, they could be modeled by a normal (Gaussian) probability distribution parameterized by the first- and second-order moments, i.e., mean velocity and turbulence intensity. However, experimental evidence (e.g., Frenkiel and Klebanoff 1967; Nakagawa and Nezu 1977; Durst et al. 
1987) has shown that turbulent velocities are non-Gaussian distributed. Rather, they are correlated with each other both spatially and temporally, revealed by their coherent (ordered) flow structures. A number of investigators (e.g., Frenkiel and Klebanoff 1973; Durst et al. 1987; Nezu and Nakagawa 1993) have suggested that higher-order moments must be included in the probability distribution to take into account such correlations. The third-order moment (skewness factor), a measure of asymmetry of the probability distribution, is a parameter used to describe the relative importance of particular burst events; the fourth-order moment (flatness or kurtosis factor) is related to the intermittency of turbulent bursting (Dittrich et al. 1996). A number of nonGaussian probability distributions have been employed to model the turbulent fluctuations, such as the lognormal distribution $(\mathrm{Wu}$ and Lin 2002; Wu and Chou 2003a), hyperbolic distribution (Durst et al. 1987), truncated Gram-Charlier distributions (Frenkiel and Klebanoff 1967; Nakagawa and Nezu 1977; Raupach 1981), and seven-parameter general distribution (BarndorffNielsen 1979; Durst et al. 1987). These distributions have been used to describe the instantaneous velocity or Reynolds stress in different subregions of the turbulent boundary layer.

Turbulent bursting events can be classified into four quadrants by their streamwise and vertical velocity fluctuations $u^{\prime}$ and $v^{\prime}$ (e.g., Robinson 1991), i.e., the outward interactions $\left(u^{\prime}>0, v^{\prime}>0\right)$, ejections $\left(u^{\prime}<0, v^{\prime}>0\right)$, inward interactions $\left(u^{\prime}<0, v^{\prime}<0\right)$, and sweeps $\left(u^{\prime}>0, v^{\prime}<0\right)$. Conventionally, ejections and sweeps were considered as most responsible for the entrainment and transport of sediment since both contribute positively to the instantaneous Reynolds stress $\left(=-\rho u^{\prime} v^{\prime}\right)$ and thus the mean bed shear stress. However, an increasing number of observations indicate that Reynolds stress is not the most relevant factor to the entrainment and transport of sediment. Rather, sediment entrainment and bedload transport are highly correlated to the instantaneous streamwise velocity (Williams et al. 1989; Nelson et al. 1995; Papanicolaou et al. 2001; Schmeeckle and Nelson 2003). Thus in this study we employ a non-Gaussian probability distribution of near-bed instantaneous streamwise velocity, rather than a joint pdf of streamwise and vertical velocities, to account for the effect of turbulent bursting.

It has been shown that the fourth-order Gram-Charlier probability density function (GC pdf) describes satisfactorily well the streamwise velocity fluctuations in the turbulent boundary layer (e.g., van Atta and Yeh 1970; Frenkiel and Klebanoff 1973; Durst et al. 1987). Moreover, it includes the higher-order correlation terms needed to account for the effect of turbulent bursting. The fourth-order GC pdf of the velocity fluctuation is given by

$$
\begin{aligned}
f_{\mathrm{GC} 4}(U)= & \frac{\exp \left(-U^{2} / 2\right)}{\sqrt{2 \pi}}\left[1+\frac{S_{u}}{3 !}\right. \\
& \left.\times\left(U^{3}-3 U\right)+\frac{F_{u}-3}{4 !}\left(U^{4}-6 U^{2}+3\right)\right]
\end{aligned}
$$

where $U=u^{\prime} / \sigma_{u}=$ normalized velocity fluctuation, in which $u^{\prime}$ $=u_{b}-\bar{u}_{b} ; u_{b}=$ near-bed instantaneous streamwise velocity; $\bar{u}_{b}$ $=$ mean approaching velocity to a sediment particle; $\sigma_{u}$ =standard deviation of $u^{\prime}$ (=turbulence intensity); $S_{u}=\left\langle u^{\prime 3}\right\rangle / \sigma_{u}^{3}$ =skewness factor of $u^{\prime}$; here \langle\rangle denotes ensemble mean; $F_{u}$ $=\left\langle u^{\prime 4}\right\rangle / \sigma_{u}^{4}=$ flatness factor of $u^{\prime}$. Eq. (1) would reduce to a normal distribution as the Gaussian skewness and flatness factors $\left(S_{u}=0\right.$ and $\left.F_{u}=3\right)$ are used. To model the near-bed streamwise velocity fluctuations with Eq. (1), four parameters (i.e., mean velocity $\bar{u}_{b}$ and higher-order moments $\sigma_{u}, S_{u}, F_{u}$ ) are required. Determination of these parameters is described below.

\section{Mean Velocity}

Nikora et al. (2001) have experimentally demonstrated that the near-bed region can be subdivided into two layers [longitudinal section shown in Fig. 1(a)], namely, the roughness layer in which the mean velocity is linearly distributed, and the logarithmic layer in which the mean velocity follows a logarithmic variation. The roughness layer covers the interfacial region between bed and flow, whose thickness $\delta$ (measured from the velocity origin) is equal to the sand diameter $D$ for uniform sand beds but is equal to $1.5 D_{50}$ for mixed-size gravel beds (Nikora et al. 2001). The origin of the linear velocity $(y=0)$ is located at a distance of $0.25 D_{84}$ below the mean bed surface (van Rijn 1984; Cheng and Chiew 1998; $\mathrm{Wu}$ and Chou 2003a), here the scaling size $D_{84}$ $\approx$ thickness of the bed surface layer (Wilcock et al. 1996; Wu and Chou 2003b). The mean velocity profile in the roughness layer can be expressed as

$$
\frac{\bar{u}}{u_{*}}=C\left(\frac{y}{\delta}\right)
$$

where $\bar{u}=$ temporal mean velocity at a height $y ; u_{*}=$ bed shear velocity $=\sqrt{\tau_{0} / \rho}, \tau_{0}=$ bed shear stress, $\rho=$ density of fluid; $C$ is evaluated with a regression relation derived from the data given in Nikora et al. (2001), i.e., $C=-0.993 \ln \left(k_{s}^{+}\right)+12.36$ for $k_{s}^{+}$ $\leqslant 1,000$, and $C=5.5$ for $k_{s}^{+}>1,000$, in which $k_{s}^{+}=$roughness Reynolds number $=u_{*} k_{s} / v$ (hereinafter + is used for quantities normalized with respect to viscous units $\left.v / u_{*}\right), k_{s}=$ equivalent roughness of Nikuradse $=2 D_{50}$ (e.g., Bridge and Bennett 1992; Ling 1995; Wu and Chou 2003a), $v=$ kinematic viscosity of fluid. Distribution of the mean velocity in the logarithmic layer can be described by

$$
\frac{\bar{u}}{u_{*}}=\frac{1}{\kappa} \ln \left(\frac{y}{y_{0}}\right)
$$

where $\kappa=$ von Karman constant $=0.4$ for clear water; $y_{0}$ is a virtual zero-velocity level. At $y=\delta$, an identical value of velocity is given by Eqs. (2) and (3), which is used to eliminate $y_{0}$ in Eq. (3) and derive a revised expression for the logarithmic velocity profile, i.e.,

$$
\frac{\bar{u}}{u_{*}}=C+\frac{1}{\kappa} \ln \left(\frac{y}{\delta}\right)
$$

Eqs. (2) and (4) are used to calculate $\bar{u}(y)$ for $y \leqslant \delta$ and $y \geqslant \delta$, respectively. For a spherical particle of size $D_{i}$ resting on the bed with a height $\Delta_{j}$ exposed to the flow [Fig. 1(b)], the area-averaged approaching velocity over the exposed frontal area $A$ is determined by

$$
\begin{aligned}
\bar{u}_{b}= & \frac{\int_{A} \bar{u} d A}{\int_{A} d A} \\
= & \frac{\int_{y_{1}}^{y_{2}} \bar{u}(y) \sqrt{\left(0.5 D_{i}\right)^{2}-\left(y-\Delta_{j}-0.25 D_{84}+0.5 D_{i}\right)^{2}} d y}{\int_{y_{1}}^{y_{2}} \sqrt{\left(0.5 D_{i}\right)^{2}-\left(y-\Delta_{j}-0.25 D_{84}+0.5 D_{i}\right)^{2}} d y}
\end{aligned}
$$

where $y_{1}=0.25 D_{84}$ and $y_{2}=0.25 D_{84}+\Delta_{j}$. Since Eq. (5) is derived 
(a)

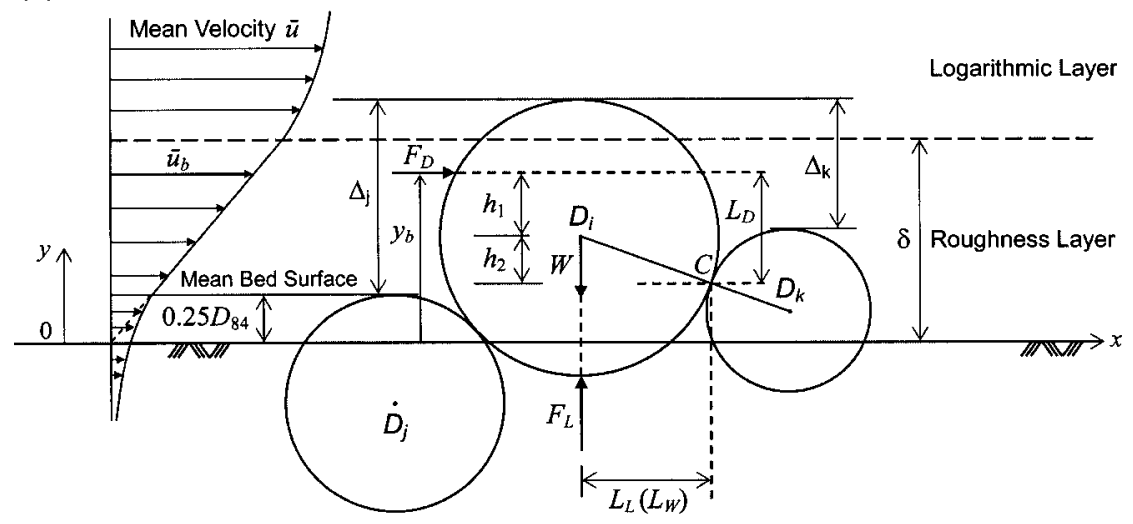

(b)

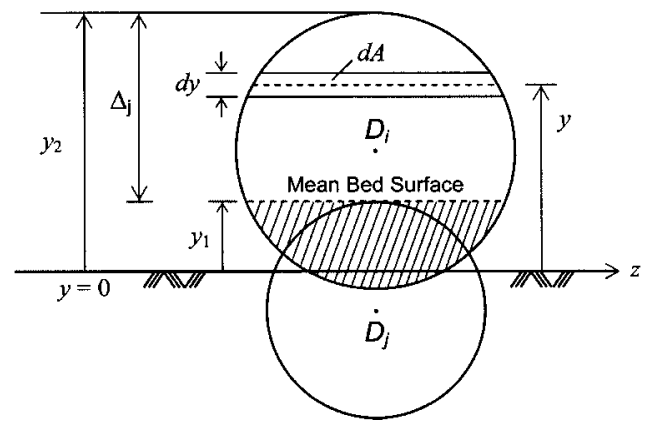

Fig. 1. (a) Longitudinal section ( $x-y$ plane) of near-bed region showing the vertical distribution of mean velocity, bed configuration of mixed-size sediment, and external forces acting on a protruding particle $D_{i}$. (b) Transverse section $\left(y-z\right.$ plane) of a protruding particle $D_{i}$ (view into flow).

for a specific $\Delta_{j}$, the mean approaching velocity $\bar{u}_{b}$ is a function of the random variable $\Delta_{j}$. The location of $\bar{u}_{b}\left[=y_{b}\right.$, as illustrated in Fig. 1(a)] can be determined with Eq. (2) for $\bar{u}_{b} \leqslant u_{*} C$, which results in $y_{b}=\bar{u}_{b} \delta / u_{*} C$; while for $\bar{u}_{b} \geqslant u_{*} C$, Eq. (4) can be used to solve for $y_{b}=\delta \exp \left[\kappa\left(\bar{u}_{b} / u_{*}-C\right)\right]$. Because $y_{b}$ is determined from $\bar{u}_{b}$, it is also a function of $\Delta_{j}$ and will be used later in derivation of the rolling threshold.

\section{Higher-Order Moments}

The near-bed coherent flow structures vary as a highly sensitive function of location and bed roughness (e.g., Grass 1971; Raupach 1981; Durst et al. 1987; Dittrich et al. 1996; Grass and Mansour-Tehrani 1996). Generally, the turbulence intensity $\sigma_{u}$ increases with the height $y^{+}(=u * y / \nu)$ in the vicinity of the bed surface, reaches its maximum value at a distance from the bed surface, and then decreases with $y^{+}$; the skewness factor $S_{u}$ is positive (i.e., sweeps dominate) in the vicinity of the bed surface, but then becomes negative (i.e., ejections become dominant) as $y^{+}$ increases; the flatness factor $F_{u}$ decreases with $y^{+}$in the vicinity of the bed surface, reaches its minimum value at a distance from the bed surface and then increases with $y^{+}$. For flows over smooth beds $\left(k_{s}^{+}<3\right)$, the maximum value of $\sigma_{u}$, the change of sign of $S_{u}$, and the minimum value of $F_{u}$ consistently occur at a height of $y_{c}^{+} \approx 13$ (Durst et al. 1987; Dittrich et al. 1996), here $y_{c}^{+}$denotes a representative coherent height at which the turbulence intensity is maximal, the contributions of sweeps and ejections are equal, and the bursting intermittency factor $\left(\propto 1 / F_{u}\right)$ is also maximal. However, for flows over transitional and rough beds $\left(k_{s}^{+}>3\right)$, a consistent location for maximum $\sigma_{u}, S_{u}=0$, and minimum $F_{u}$ cannot
Table 1. Compilation of Published Experimental and Simulation Data Used for Analysis of Near-bed Coherent Flow Structures

\begin{tabular}{lcc}
\hline Source & Method/fluid & Roughness \\
\hline $\begin{array}{l}\text { Grass (1971) } \\
\text { Kreplin and Eckelmann }\end{array}$ & Hydrogen-bubble/water & Transitional \\
(1979) & Hot-film/oil & Smooth \\
Andreopoulos and Bradshaw & Hot-wire/air & Fully rough \\
(1981) & & \\
Raupach (1981) & Hot-wire/air & Fully rough \\
Johansson and Alfredsson & Hot-film/water & Smooth \\
(1982) & & \\
Andreopoulos et al. (1984) & Hot-wire/air & Smooth \\
& & Transitional \\
Alfredsson et al. (1988) & Hot-wire/air & Smooth \\
& Hot-film/oil & \\
& Hot-film/water & \\
Dittrich et al. (1996) & LDA/water & Fully rough \\
Di Cicca et al. (2002) & PIV/water & Transitional \\
Djendidi et al. (1999) & LDV/water & Smooth \\
Spalart (1988) & DNS & Transitional \\
Moin and Kim (1982) & LES & Transitional \\
Kim et al. (1987) & DNS & Smooth \\
Rodi et al. (1993) & DNS & Transitional \\
Cui et al. (2003) & LES & Transitional \\
& & Fully rough \\
\hline Note: LDA & &
\end{tabular}

Note: $\mathrm{LDA}=$ laser Doppler anemometer; $\mathrm{LDV}=$ laser Doppler velocimetry; PIV=particle image velocimetry; DNS $=$ direct numerical simulation; and LES = large eddy simulation. 


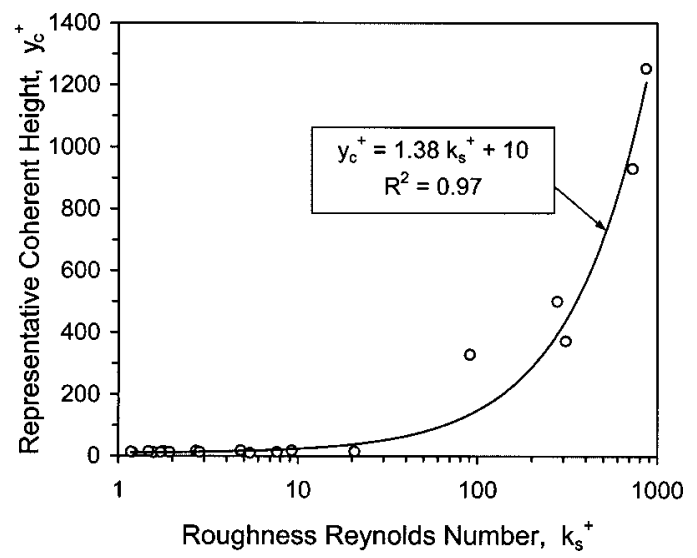

Fig. 2. Relation between representative coherent height and roughness Reynolds number

be found. Thus, for the transitional and rough beds, the height of maximum $\sigma_{u}$, rather than the height of $S_{u}=0$ or minimum $F_{u}$, is taken to be $y_{c}^{+}$. Based on a compilation of the published experimental and simulation data for a wide range of bed roughness (listed in Table 1), we found that $y_{c}^{+}$increases linearly with $k_{s}^{+}$ (shown in Fig. 2). The higher-order moments at $y_{c}^{+}$are further extracted from these compiled data and used to develop a set of quantitative relations (demonstrated in Fig. 3), expressed as follows for the smooth and transitional beds:

$$
\begin{gathered}
\sigma_{u} / u_{*}=-0.187 \ln \left(k_{s}^{+}\right)+2.93 \\
S_{u}=0.102 \ln \left(k_{s}^{+}\right) \text {for } k_{s}^{+} \leqslant 70 \\
F_{u}=0.136 \ln \left(k_{s}^{+}\right)+2.30
\end{gathered}
$$

For the fully rough beds, the higher-order moments appear to be independent of $k_{s}^{+}$, i.e.,

$$
\sigma_{u} / u_{*}=2.14
$$

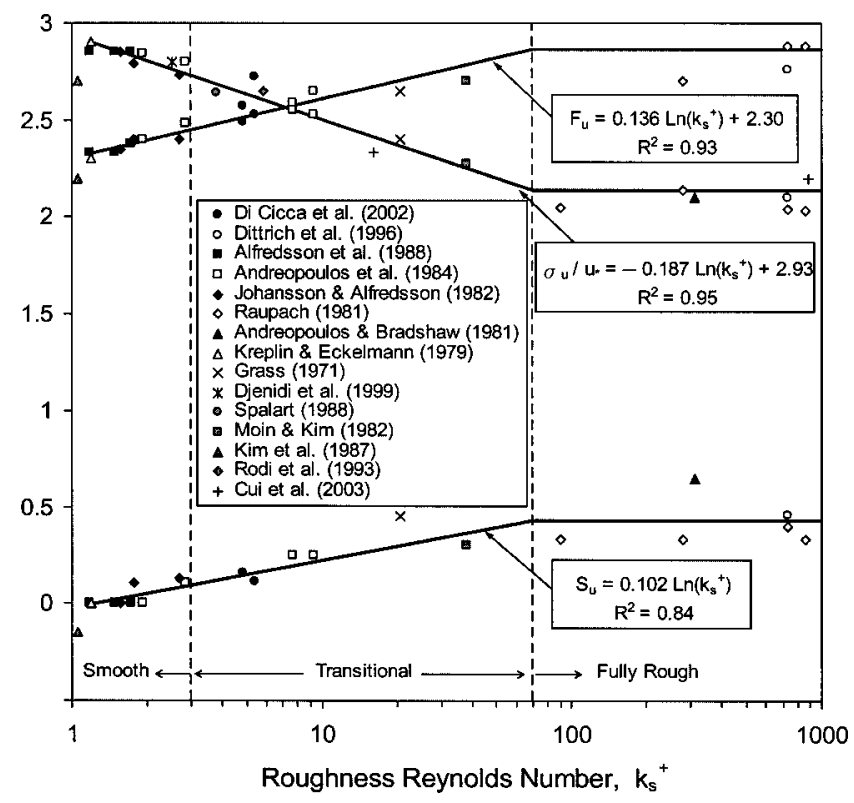

Fig. 3. Variations of second-, third-, and fourth-order moments of longitudinal velocity fluctuation with roughness Reynolds number

$$
S_{u}=0.43 \text { for } k_{s}^{+}>70
$$

$$
F_{u}=2.88
$$

Eqs. (5) and (6) can be used to determine the mean velocity and higher-order moments required in Eq. (1) for specifying $f_{\mathrm{GC} 4}(U)$ or equivalently the probability distribution of near-bed instantaneous streamwise velocity, which is given by

$$
f_{u}\left(u_{b}\right)=\frac{1}{\sigma_{u}} f_{\mathrm{GC} 4}(U)=\frac{1}{\sigma_{u}} f_{\mathrm{GC} 4}\left(\frac{u_{b}-\bar{u}_{b}}{\sigma_{u}}\right)
$$

\section{Bed Configuration of Mixed-Size Sediment}

Consider a spherical particle of size $D_{i}$ resting on the bed composed of mixed-size sediment [Fig. 1(a)]. This particle $D_{i}$ is in contact with an upstream particle of size $D_{j}$ and a downstream particle of size $D_{k}$. For natural water-worked sediments, the random bed configuration is typically described by the protrusion height and friction angle (Kirchner et al. 1990; Buffington et al. 1992). Herein the protrusion height of particle $D_{i}$ is represented by its exposure height $\Delta_{j}$ above particle $D_{j}$; the friction angle between particles $D_{i}$ and $D_{k}$ is represented by a friction height $\Delta_{k}$ above particle $D_{k}$ [Fig. 1(a)]. Both $\Delta_{j}$ and $\Delta_{k}$ are random variables, accompanied by random combinations of $D_{j}$ and $D_{k}$, resulting in the hiding-exposure effect of the shear stress applied on particle $D_{i}$, which are described below.

\section{Exposure Height}

Following the previous studies (Paintal 1971; Kirchner et al. 1990; Wu et al. 2000; Wu and Chou 2003a), we assume the exposure height of particle $D_{i}$ to be uniformly distributed, with its upper and lower limits $=D_{i}$ and 0 , respectively. The pdf of $\Delta_{j}$ can be expressed as

$$
f_{\mathrm{EH}}\left(\Delta_{j}\right)=1 / D_{i} \text { for } 0 \leqslant \Delta_{j} \leqslant D_{i}
$$

\section{Friction Height}

The pdf of friction height is not available from the literature. Nonetheless, measurements show that the friction angles of individual grains are uncorrelated to their protrusion heights, and that the friction angle is quasi-uniformly distributed in the range between $\sim 10$ and $80^{\circ}$ (Kirchner et al. 1990). Accordingly, the pdf of $\Delta_{k}$ is also assumed to follow a uniform distribution, with its upper and lower limits taken to be $D_{i}$ and 0 , respectively, i.e.,

$$
f_{\mathrm{FH}}\left(\Delta_{k}\right)=1 / D_{i} \text { for } 0 \leqslant \Delta_{k} \leqslant D_{i}
$$

\section{Hiding Factor}

The hiding factor approach is useful to account for the hidingexposure effect of mixed-size sediment on the applied shear stress. The effective shear stress applied on the coarser particle is greater than the shear stress evaluated from the mean bed roughness, whereas the effective shear stress applied on the finer particle is smaller than the mean bed shear stress (e.g., Proffitt and Sutherland 1983; Misri et al. 1984; Sun and Donahue 2000), which can be expressed as 


$$
\tau_{i}=\xi_{i} \tau_{0}
$$

where $\tau_{i}$ =effective shear stress applied on particles $D_{i} ; \tau_{0}$ =mean bed shear stress; $\xi_{i}=$ hiding factor for particles $D_{i}$, which can be evaluated with the following probabilistic approach (Wu et al. 2000):

$$
\xi_{i}=\left(P_{e i} / P_{h i}\right)^{0.6}
$$

here $P_{e i}$ and $P_{h i}=$ total exposed and hidden probabilities of particles $D_{i}$, as determined by

$$
\begin{gathered}
P_{e i}=\sum_{j=1}^{N} p_{j} \frac{D_{i}}{D_{i}+D_{j}} \\
P_{h i}=\sum_{j=1}^{N} p_{j} \frac{D_{j}}{D_{i}+D_{j}}
\end{gathered}
$$

where $p_{j}=$ proportion of particles $D_{j}$ in the bed material; $N$ $=$ total number of grain-size fractions, such that $\sum_{j=1}^{N} p_{j}=1$. Eq. (11b) also implies that $P_{e i}+P_{h i}=1$. For unisize sediment, it follows that $P_{e i}=P_{h i}=0.5$ and thus $\xi_{i}=1$.

\section{Formulation of Entrainment Probability}

Shown in Fig. 1(a) are the external forces acting on a particle $D_{i}$, including the submerged weight, $W$, and the instantaneous hydrodynamic forces, which can be resolved into an effective drag force, $F_{D}$, and an effective lift force, $F_{L}$. The following expressions have been used for these forces (e.g., Cheng and Chiew 1999; Fischer et al. 2002; Wu and Chou 2003a):

$$
\begin{gathered}
W=\left(\gamma_{s}-\gamma\right) \frac{\pi D_{i}^{3}}{6} \\
F_{D}=\xi_{i} C_{D} \frac{\rho A u_{b}^{2}}{2} \\
F_{L}=\xi_{i} C_{L} \frac{\rho A u_{b}^{2}}{2}
\end{gathered}
$$

where $\gamma_{s}$ and $\gamma=$ specific weights of sediment and water, respectively; $A=$ frontal area exposed to the flow [=denominator of Eq. (5)]; $u_{b}=$ near-bed instantaneous streamwise velocity; $C_{D}$ $=\left(24 / R_{p}\right)\left(1+0.15 R_{p}^{0.687}\right)=$ Stokes drag coefficient, valid for $R_{p}$ $\leqslant 1,754, \mathrm{R}_{p}=$ particle Reynolds number, defined by $\bar{u}_{b} \Delta_{j} / \nu$, while $C_{D}=0.36$ for $\mathrm{R}_{p}>1,754$. The use of $\bar{u}_{b}$ for evaluating $C_{D}$ is a necessarily simplified treatment (Wu and Chou 2003a). Much less is known about the lift coefficient $C_{L}$ than about $C_{D}$. Based on the measurements of lift-drag ratio (Patnaik et al. 1994), it has been inferred that $C_{L} / C_{D} \approx 1$ for $\mathrm{R}_{p}<8,000$ (Wu and Chou 2003a), which is used herein to determine $C_{L}$.

\section{Entrainment Thresholds}

\section{Rolling Threshold}

For a resting particle $D_{i}$ that is in contact with a downstream particle $D_{k}$ at point $C$ [as illustrated in Fig. 1(a)], the threshold condition for rolling of particle $D_{i}$ to occur is that the instantaneous turning moment exceeds the resisting moment, which can be expressed by

$$
F_{D} L_{D}+F_{L} L_{L}>W L_{W}
$$

in which $L_{D}, L_{L}$, and $L_{W}=$ the moment arms (about point $C$ ) of $F_{D}$, $F_{L}$, and $W$, respectively. Combining Eqs. (12) and (13) gives

$$
u_{b}^{2}>B_{R}^{2}
$$

where $B_{R}$ denotes the rolling threshold, as expressed by

$$
B_{R}=\sqrt{\frac{2 L_{W}}{C_{D} L_{D}+C_{L} L_{L}} \frac{\pi D_{i}^{3}}{6 A} \frac{\gamma_{s}-\gamma}{\rho \xi_{i}}}=u * \sqrt{\frac{2 L_{W}}{C_{D} L_{D}+C_{L} L_{L}} \frac{\pi D_{i}^{2}}{6 A} \frac{1}{\theta_{i}}}
$$

where $\theta_{i}=\tau_{i} /\left(\gamma_{s}-\gamma\right) D_{i}=$ dimensionless effective shear stress of particle $D_{i}$. For uniform sediment of size $D$ (hiding factor $=1$ ), $\theta_{i}$ reduces to $\theta=\tau_{0} /\left(\gamma_{s}-\gamma\right) D$. The particle $D_{i}$ will start to roll when the inequality in Eq. (14) is satisfied.

The submerged weight $W$ acts on the center of particle $D_{i}$ [Fig. 1(a)]; the lift force $F_{L}$ is perpendicular to the flow direction and acts on a line passing through the center of particle $D_{i}$. The drag force $F_{D}$ is parallel to the flow direction, and has been assumed to act on the height $y_{b}$ where the mean velocity $\bar{u}=\bar{u}_{b}$ (Wu and Chou 2003a), which leads to

$$
L_{D}=h_{1}+h_{2}
$$

where $\quad h_{1}=y_{b}-0.25 D_{84}-\Delta_{j}+0.5 D_{i}, \quad$ and $\quad h_{2}=D_{i}\left(\Delta_{k}+0.5 D_{k}\right.$ $\left.-0.5 D_{i}\right) /\left(D_{i}+D_{k}\right)$. The identical value of $L_{L}$ and $L_{W}$ can be expressed as

$$
L_{L}=L_{W}=\sqrt{\left(0.5 D_{i}\right)^{2}-h_{2}^{2}}
$$

Eqs. (16) and (17) imply that the moment $\operatorname{arm} L_{D}$ varies with $D_{k}$, $\Delta_{j}$, and $\Delta_{k}$, while the moment $\operatorname{arm} L_{L}\left(\right.$ or $\left.L_{W}\right)$ is dependent on $D_{k}$ and $\Delta_{k}$. Accordingly, $B_{R}$ is also a function of the random variables $D_{k}, \Delta_{j}$, and $\Delta_{k}$.

\section{Lifting Threshold}

The threshold condition for lifting of particle $D_{i}$ to occur is that the instantaneous lift force acting on particle $D_{i}$ exceeds its submerged weight, as expressed by

$$
F_{L}>W
$$

Using the expressions for $F_{L}$ and $W$ given in Eq. (12), one can rewrite Eq. (18) as

$$
u_{b}^{2}>B_{L}^{2}
$$

where $B_{L}$ denotes the lifting threshold, which can be expressed as

$$
B_{L}=\sqrt{\frac{2}{C_{L}} \frac{\pi D_{i}^{3}}{6 A} \frac{\gamma_{s}-\gamma}{\rho \xi_{i}}}=u_{*} \sqrt{\frac{2}{C_{L}} \frac{\pi D_{i}^{2}}{6 A} \frac{1}{\theta_{i}}}
$$

The particle $D_{i}$ will start to move in the lifting mode when the criterion given in Eq. (19) is met. Eq. (20) implies that $B_{L}$ is only a function of the random variable $\Delta_{j}$. It is also known from Eqs. (15) and (20) that $B_{R} / B_{L}=\sqrt{L_{L} /\left(L_{D}+L_{L}\right)}<1$, indicating that $B_{L}>B_{R}$ (Ling 1995; Wu and Chou 2003a). With the rolling and lifting thresholds established above and the pdf of instantaneous velocity, the entrainment probabilities can be precisely formulated as follows.

\section{Entrainment Probabilities}

\section{Rolling Probability}

The probability of entrainment in the pure rolling mode (i.e., not lifted off the bed) can be expressed as 
$P_{R}=P\left(B_{R}^{2}<u_{b}^{2}<B_{L}^{2}\right)=P\left(-B_{L}<u_{b}<-B_{R}\right)+P\left(B_{R}<u_{b}<B_{L}\right)$

With the pdf of $u_{b}$ given in Eq. (7), one can rewrite Eq. (21) as the following form:

$$
P_{R}=\int_{-B_{L}}^{-B_{R}} f_{u}\left(u_{b}\right) d u_{b}+\int_{B_{R}}^{B_{L}} f_{u}\left(u_{b}\right) d u_{b}
$$

The rolling probability obtained from Eq. (22) is for a specific set of $\left(D_{k}, \Delta_{j}, \Delta_{k}\right)$. Thus the mean rolling probability of particle $D_{i}$, denoted as $P R$, is the expected value of Eq. (22) over the full ranges of these three random variables. Given that $D_{k}, \Delta_{j}$, and $\Delta_{k}$ are independent random variables, $P R$ can be expressed as

$$
P R=\int_{0}^{D_{i}}\left\{\int_{0}^{D_{i}}\left[\int_{D_{\min }}^{D_{\max }} P_{R} f_{s}\left(D_{k}\right) d D_{k}\right] f_{\mathrm{FH}}\left(\Delta_{k}\right) d \Delta_{k}\right\} f_{\mathrm{EH}}\left(\Delta_{j}\right) d \Delta_{j}
$$

where $f_{s}\left(D_{k}\right)=$ pdf of downstream grain size $D_{k} ; D_{\min }$ and $D_{\max }$ $=$ lower and upper bounds of size distribution. For a sediment mixture divided into $N$ discrete size fractions, the innermost term of Eq. (23), $\int_{D_{\min }}^{D_{\max }} P_{R} f_{s}\left(D_{k}\right) d D_{k}$, can be replaced by $\sum_{n=1}^{N} p_{n} P_{R}\left(D_{n}\right)$, here $p_{n}$ and $D_{n}$ are the proportion and representative diameter of the $n$th size fraction, respectively. Using Eqs. (8) and (9), one can further rewrite Eq. (23) as

$$
P R=\frac{1}{D_{i}^{2}} \int_{0}^{D_{i}}\left\{\int_{0}^{D_{i}}\left[\sum_{n=1}^{N} p_{n} P_{R}\left(D_{n}, \Delta_{k}, \Delta_{j}\right)\right] d \Delta_{k}\right\} d \Delta_{j}
$$

Eq. (24) can be solved numerically to evaluate the mean rolling probability of particle $D_{i}$, given the flow and sediment conditions, i.e., $\tau_{0}$ and $\left(p_{n}, D_{n}\right)$ of all size fractions.

\section{Lifting Probability}

The probability of entrainment in the lifting mode (i.e., for particle $D_{i}$ to start losing contact with the bed) can be expressed as

$$
\begin{aligned}
P_{L}=P\left(B_{L}^{2}<u_{b}^{2}\right) & =P\left(-B_{L}>u_{b}\right)+P\left(B_{L}<u_{b}\right) \\
& =1-P\left(-B_{L} \leqslant u_{b} \leqslant B_{L}\right)
\end{aligned}
$$

Integrating the pdf of $u_{b}$, one can rewrite Eq. (25) as

$$
P_{L}=1-\int_{-B_{L}}^{B_{L}} f_{u}\left(u_{b}\right) d u_{b}
$$

The lifting probability calculated from Eq. (26) is for a specific value of $\Delta_{j}$. The mean lifting probability of particle $D_{i}$, denoted as $P L$, is the expected value of Eq. (26) over the full range of $\Delta_{j}$, i.e.,

$$
P L=\int_{0}^{D_{i}} P_{L}\left(\Delta_{j}\right) \cdot f_{\mathrm{EH}}\left(\Delta_{j}\right) d \Delta_{j}
$$

With Eq. (8), one can further rewrite Eq. (27) as

$$
P L=\frac{1}{D_{i}} \int_{0}^{D_{i}} P_{L}\left(\Delta_{j}\right) d \Delta_{j}
$$

Eq. (28) is also solved numerically to evaluate the mean lifting probability of particle $D_{i}$ for the specified flow and sediment conditions.

From Eqs. (21) and (25), we know that rolling and lifting are two independent modes. Thus the total entrainment probability $P_{T}$

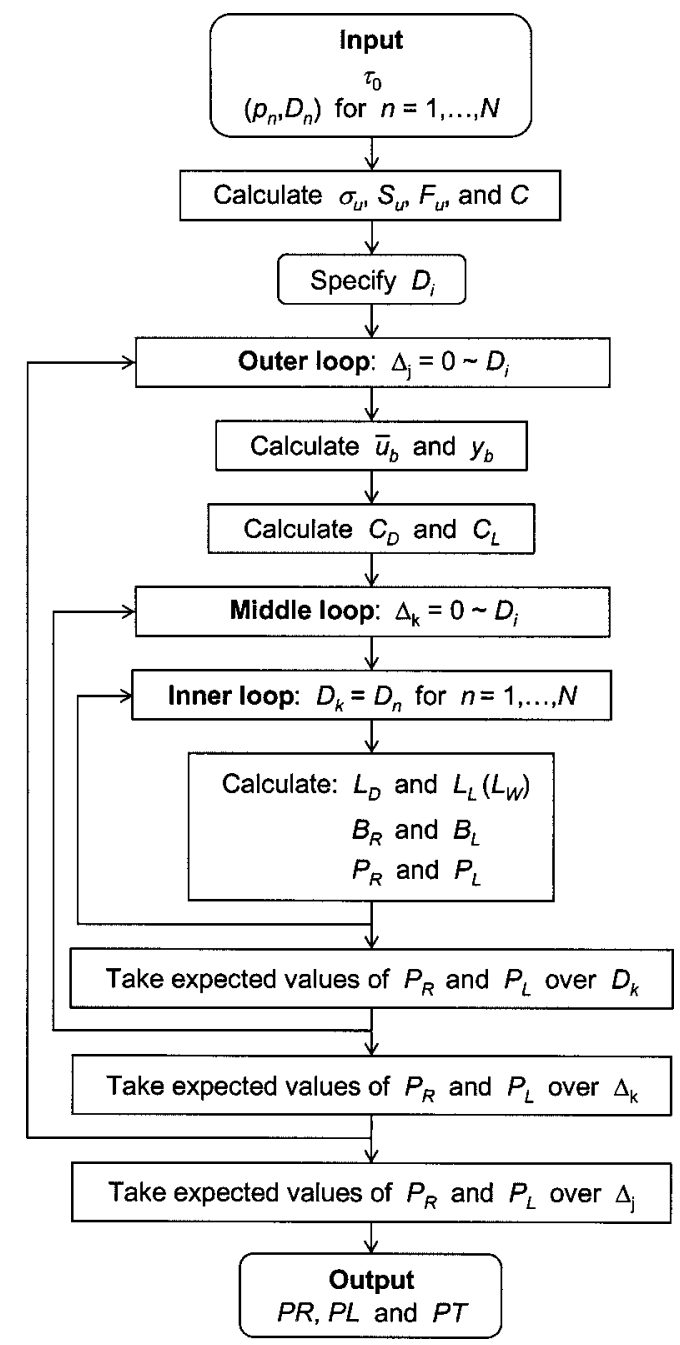

Fig. 4. Flowchart of triple-loop computation procedure of entrainment probabilities

can be evaluated by $P_{R}+P_{L}$. Taking the expected value of $P_{T}$ over the full ranges of all random variables yields the mean total entrainment probability

$$
P T=P R+P L
$$

where $P R$ and $P L$ are obtained from Eqs. (24) and (28), respectively.

\section{Computation Procedure}

Computation of the entrainment probabilities is implemented with a triple-loop procedure. The flowchart of the computation procedure is illustrated in Fig. 4, where the outer loop is for the full range of exposure height $\Delta_{j}$, the middle loop is for the full range of friction height $\Delta_{k}$, and the inner loop is for the full range of downstream grain size $D_{k}$. The required input data include the mean bed shear stress $\tau_{0}$ and $\left(p_{n}, D_{n}\right)$ of all size fractions, which are used to calculate $u_{*}$ and interpolate $D_{50}$ and $D_{84}$, respectively, and thus $k_{s}^{+}$can be evaluated. Based on the $k_{s}^{+}$value, the higherorder moments $\sigma_{u}, S_{u}$, and $F_{u}$, and the coefficient $C$ of the linear velocity profile can be determined. The particle size of interest, $D_{i}$, is then specified and used to calculate $\xi_{i}$ and $\theta_{i}$. For a given $\Delta_{j}$ in the outer loop, $\bar{u}_{b}, y_{b}$, and the corresponding $C_{D}\left(C_{L}\right)$ can be evaluated, which are coupled with a given $\Delta_{k}$ in the middle loop 


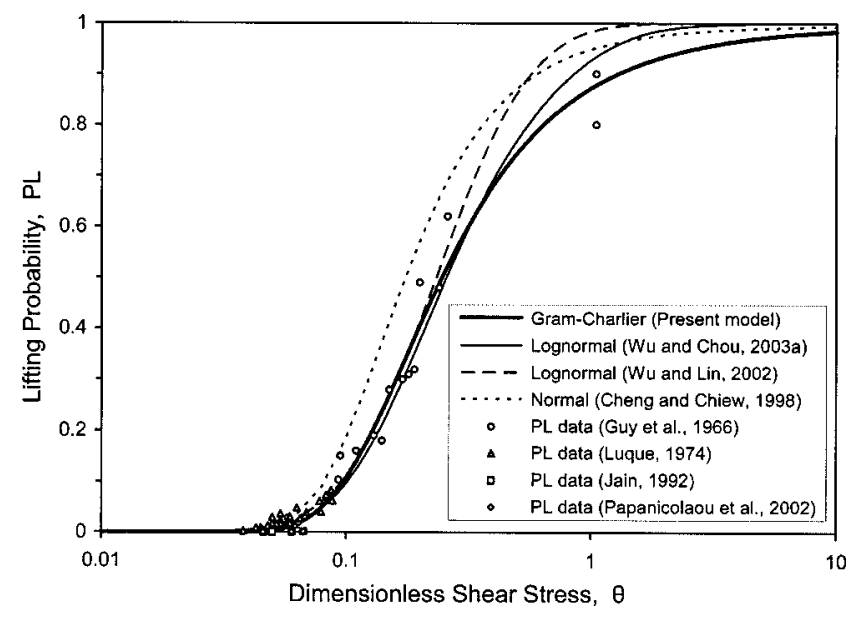

Fig. 5. Variations of lifting probability with dimensionless shear stress for unisize sediments (comparison of model results and published data)

and a $D_{k}$ in the inner loop to calculate $L_{D}$ and $L_{L}\left(L_{W}\right)$. Then the entrainment thresholds $B_{R}$ and $B_{L}$ are determined and used to evaluate $P_{R}$ and $P_{L}$. Through the triple-loop procedure, the expected values of $P_{R}$ and $P_{L}$ are computed over the full ranges of $D_{k}, \Delta_{k}$, and $\Delta_{j}$. The output results include the mean rolling, lifting, and total entrainment probabilities of those surface particles with the specified size $D_{i}$.

\section{Results and Discussion}

\section{Entrainment Probabilities}

Herein, the computed entrainment probabilities are verified with the published experimental data for both unisize and mixed-size sediments. In addition, the present model is compared with several previous ones to demonstrate the improvement achieved in this study.

\section{Unisize Sediment}

Variations of the lifting probability $P L$ with the dimensionless shear stress $\theta$ are demonstrated in Fig. 5, where the computed result of the present model is in good agreement with the published data for unisize sediments, including those of Guy et al.
Table 3. Euclidean Norms and Coefficients of Determination for Different Model Results

\begin{tabular}{lcc}
\hline $\begin{array}{c}\text { Entrainment probability } \\
\text { model }\end{array}$ & $\begin{array}{c}\text { Euclidean norm, } \\
\|e\|_{2}\end{array}$ & $\begin{array}{c}\text { Coefficient } \\
\text { of determination, } \\
R^{2}\end{array}$ \\
\hline Cheng and Chiew (1998) & 0.541 & 0.858 \\
Wu and Lin (2002) & 0.266 & 0.966 \\
Wu and Chou (2003a) & 0.245 & 0.971 \\
Present model & 0.209 & 0.979 \\
\hline
\end{tabular}

(1966); Luque (1974); Jain (1992); and Papanicolaou et al. (2002). The results of three previous models (Cheng and Chiew 1998; Wu and Lin 2002; Wu and Chou 2003a) are also shown in Fig. 5 for comparisons. The normal or lognormal (LN) pdf of $u_{b}$ has been used in these previous studies, among which only $\mathrm{Wu}$ and Chou (2003a) considered the random grain protrusion. A summary of these models is provided in Table 2 . The result of the present model demonstrates a significant improvement in the prediction of $P L$, especially for the high value of $\theta(\approx 1)$ at which the previous models have had a consistent tendency to overestimate $P L$. By incorporating the 4th-order GC pdf and the randomness of grain protrusion, the present model reduces the magnitudes of $P L$ corresponding to the high values of $\theta$, i.e., the effects associated with the smaller particle sizes or lower lifting thresholds are taken into account more precisely. The effect of the fourth-order GC pdf is discussed in more detail in the subsequent section. Here, to quantitatively demonstrate the agreement between the predicted and observed results, the Euclidean norm $\|e\|_{2}$ (i.e., the root-sumsquare of errors) and coefficient of determination $R^{2}$ for different models are listed in Table 3, where the $\|e\|_{2}$ value of the present model decreases 15,21 , and $61 \%$ from the $\|e\|_{2}$ values of the $\mathrm{Wu}-\mathrm{Chou}, \mathrm{Wu}-\mathrm{Lin}$, and Cheng-Chiew models, respectively, while the $R^{2}$ value of the present model increases $0.8,1.3$, and $14 \%$ from the $R^{2}$ values of the corresponding previous models. These improvements are attributable to combinations of the additional factors considered in the present study, which include the near-bed coherent structures and random grain protrusion (i.e., exposure and friction heights). However, the effect of particle hiding and exposure as well as the randomness of adjacent grain sizes do not exist for the unisize sediments.

\section{Mixed-Size Sediment}

The present model is further used to compute the total entrainment probabilities $P T$ of mixed-size sediments under the partial-

Table 2. Summary of Entrainment Probability Models

\begin{tabular}{lcc}
\hline Entrainment probability model & pdf of turbulent velocity & Factors considered \\
\hline Lifting probability (Cheng and Chiew 1998) & Normal & Unisize sediment \\
Full grain exposure & Unisize sediment \\
Lifting probability (Wu and Lin 2002) & Lognormal grain exposure & Unisize sediment \\
Rolling, lifting, and total probabilities (Wu and Chou 2003a) & Lognormal & Random grain exposure \\
Rolling, lifting, and total probabilities (present model) & Mixed-size sediment & Turbulent bursting \\
& Fourth-order Gram-Charlier & (Higher-order correlations) \\
& Random grain protrusion & (Exposure and friction heights) \\
\end{tabular}




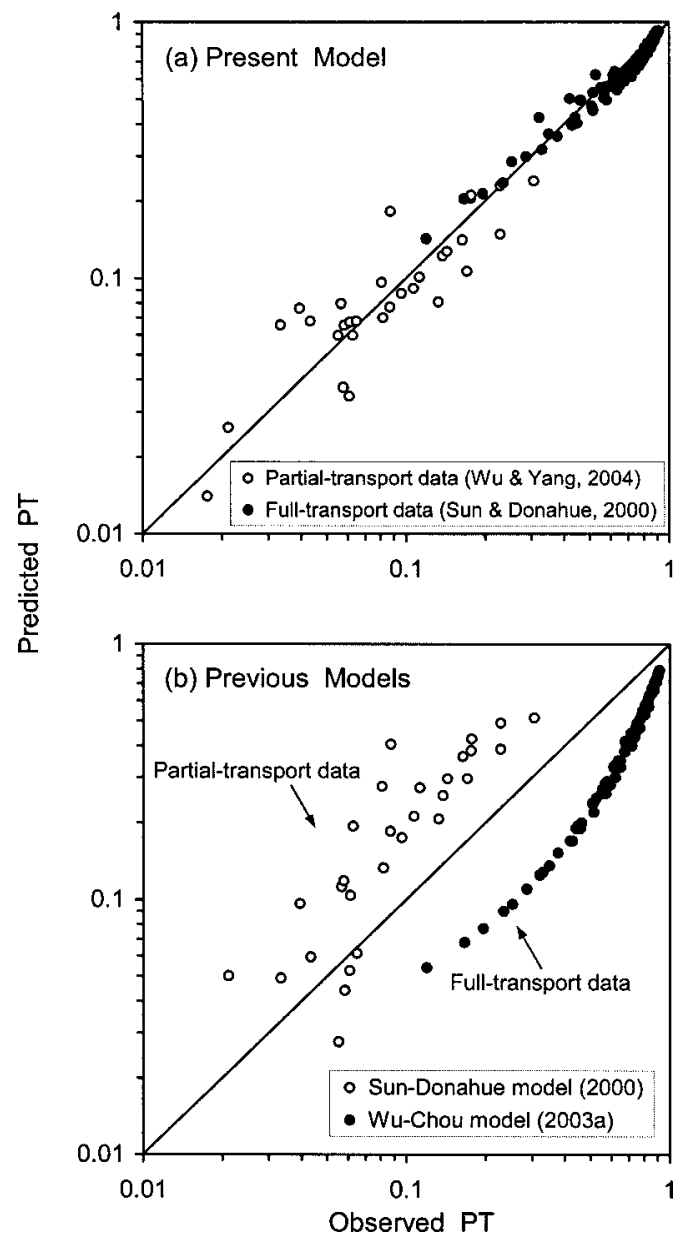

Fig. 6. Comparison of predicted and observed total entrainment probabilities for partial- and full-transport conditions of mixed-size sediments: (a) predicted with present model and (b) predicted with two previous models

and full-transport conditions. It is well known that the transport of sediment in gravel-bed rivers is associated with a condition of partial transport over a range of flows, within which some grains exposed on the bed surface are active (i.e., entrained at least once over the duration of a transport event), while the remaining are immobile. However, under the full-transport condition all surface grains are active. The partial-transport entrainment probabilities from $\mathrm{Wu}$ and Yang (2004) and the full-transport entrainment probabilities from Sun and Donahue (2000) are used for verification of the present model, the former include seven sets of data for the fully rough beds, with $k_{s}^{+}$ranging from 360 to 500, the latter include nine sets of data for the transitional beds, with $k_{s}^{+}$ ranging from 28 to 64 . A comparison of the predicted and observed $P T$ values is shown in Fig. 6(a), where good agreement between the predicted and observed results is demonstrated, with the global coefficient of determination $R^{2}=0.971$. It is also shown in Fig. 6(a) that the full-transport $P T$ values are mostly greater than the partial-transport values, with the former ranging between 0.1 and 1 (of which over $80 \%$ are greater than 0.5 ) and the latter covering the orders of $10^{-2}$ and $10^{-1}$. The results appear to indicate that the present model can be applied to evaluate the entrainment probabilities of mixed-size sediments under the partial- and full-transport conditions.

To further demonstrate the merit of the present model, we compare our results with those of two previous models, one was developed for evaluating the full transport of mixed-size sediments (Sun and Donahue 2000) and the other was for the entrainment of unisize sediments (Wu and Chou 2003a). To explore the suitability of the two previous models to the conditions beyond their original scopes, we use the Sun-Donahue model to calculate the partial-transport entrainment probabilities [data of $\mathrm{Wu}$ and Yang (2004)] and use the Wu-Chou model to compute the entrainment probabilities of mixed-size sediments [data of Sun and Donahue (2000)]. The results [shown in Fig. 6(b)] reveal that the Sun-Donahue model overestimates nearly all of the partialtransport entrainment probabilities yet the $\mathrm{Wu}-\mathrm{Chou}$ model consistently underestimates the entrainment probabilities of mixedsize sediments. The overestimation of partial-transport $P T$ values by the Sun-Donahue model is probably due to the fact that the predictive relation proposed by Sun and Donahue (2000) was derived from the best fitting to the full-transport data, while the consistent underestimation of full-transport $P T$ values of mixedsize sediments by the $\mathrm{Wu}-\mathrm{Chou}$ model is believed to originate from neglecting the grain-size nonuniformity and effect of nearbed coherent flow structures. In view of these results, it is clear that the previous models have limited applicability on the prediction of entrainment probabilities for the partially transported mixed-size sediments.

\section{Effects of Higher-Order Correlations}

In this study, we have included the higher-order moments in the probability distribution of turbulent velocity to incorporate the near-bed coherent flow structures. Frenkiel and Klebanoff (1973) have compared the experimentally determined probability density distribution of near-bed streamwise velocity with the second-, fourth-, and sixth-order GC pdfs. Their results demonstrated that both the fourth- and sixth-order GC pdfs are in good agreement with the experimentally determined probability distribution, while the second-order GC (Gaussian) pdf is not as good as the other two. The fourth-order GC pdf is inferior to the sixth-order one only at the right end of the distribution, where the probability densities are almost negligible. Herein, to investigate the effects of the higher-order correlations on flows over smooth and rough beds, we compare the truncated second-, third-, and fourth-order GC pdfs of $u_{b}$, as shown in Fig. 7 , where the mean velocity $\bar{u}_{b}$ is arbitrarily taken as $2 \mathrm{~cm} / \mathrm{s}$. For the smooth bed, we select $k_{s}^{+}=3$ and $k_{s}=2 \mathrm{~mm}$, while for the fully rough bed, we use $k_{s}^{+}=100$ and $k_{s}=20 \mathrm{~mm}$. From these specified numbers, the values of $\sigma_{u}, S_{u}$, and $F_{u}$ can be determined and then used to calculate $f_{u}\left(u_{b}\right)$. The results reveal that for the smooth bed, the second- and third-order GC pdfs are similar to each other but different from the fourthorder one. However, for the fully rough bed, the third- and fourthorder GC pdfs appear to be identical. As mentioned earlier, the second-order GC pdf is in fact a normal distribution defined by two parameters $\bar{u}_{b}$ and $\sigma_{u}$. For smooth beds, $S_{u}$ is nearly equal to 0 (Fig. 3) such that the third-order correlation term is ineffective and thus the third-order GC pdf reduces to a normal distribution. For fully rough beds, $F_{u}(=2.88)$ is very close to the Gaussian value $(=3)$, thus the fourth-order correlation term is almost vanishing and the fourth-order GC pdf approaches a third-order one. These results imply that for fully rough beds, the third-order GC pdf can be used to approximate the fourth-order one. However, for smooth beds, the fourth-order GC pdf should be used to incorporate the more complete effect of higher-order correlations.

It is also shown in Fig. 7 that the standard deviation of $f_{u}\left(u_{b}\right)$ for the smooth bed is smaller than that for the rough bed, with the rough-bed $\sigma_{u}$ being 2.6 times the smooth-bed value, indicating 

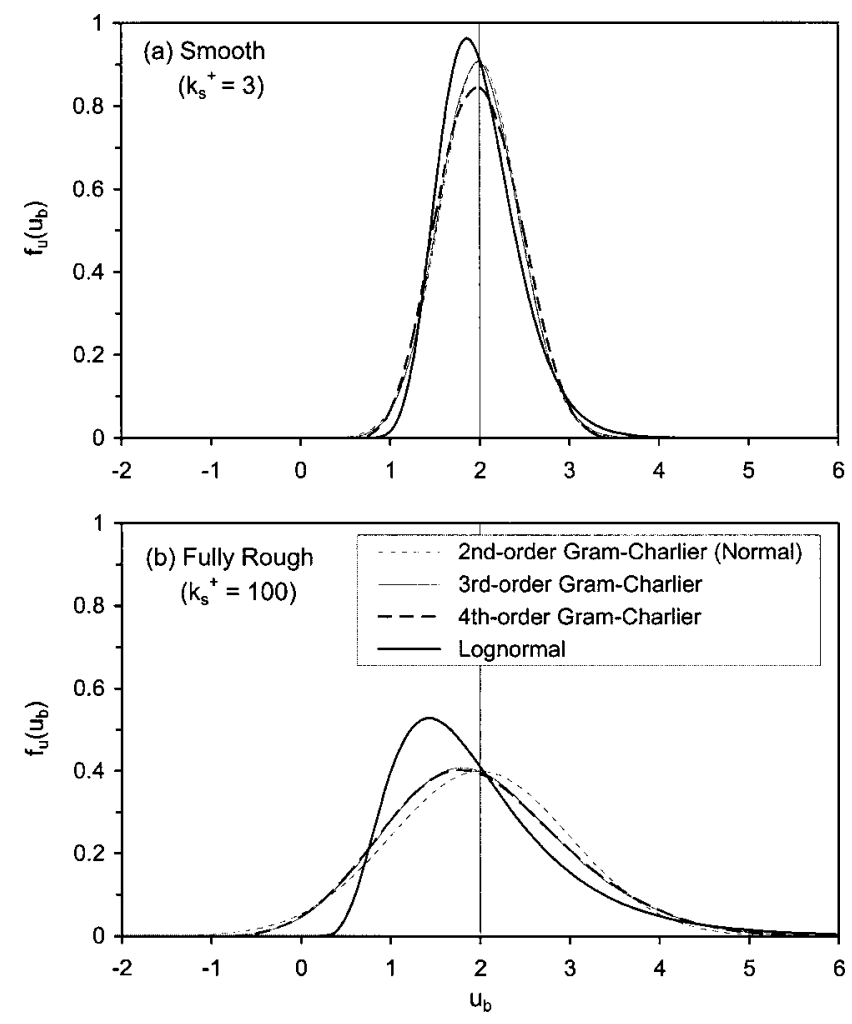

Fig. 7. Comparison of truncated second-, third-, and fourth-order Gram-Charlier and lognormal pdfs of $u_{b}$ for (a) smooth and (b) fully rough beds (note that second-order Gram-Charlier pdf=normal pdf)

that for the rough beds the magnitudes of near-bed velocity fluctuations (or turbulence intensity) are significantly greater. For the smooth bed, the primary difference between the fourth-order GC pdf and normal pdf is in the kurtosis, with the normally distributed $u_{b}$ more concentrated in the vicinity of $\bar{u}_{b}$; while for the fully rough bed, the primary difference between the fourth-order GC pdf and normal pdf is in the skewness, with the fourth-order GC pdf more skewed to the left. Thus the conventional use of normal pdf for turbulent velocity would result in an overestimation of kurtosis for the smooth beds but an underestimation of skewness for the rough beds. To further investigate the distribution effect, the LN pdfs (with the $\bar{u}_{b}$ and $\sigma_{u}$ identical to the values specified to the GC pdfs) are also illustrated in Fig. 7. It is revealed that both the skewness and kurtosis of the LN pdfs are higher than those of the corresponding GC pdfs, leading to an overestimation of $f_{u}\left(u_{b}\right)$ in the lower near-mean region but an underestimation in the far lower region, and an underestimation in the upper near-mean region but an overestimation in the far upper region. The discrepancies between the observed and predicted $P L$ values with the previous lognormal models (Wu and Lin 2002; Wu and Chou 2003a), as shown in Fig. 5, are believed to arise from such higher skewness and kurtosis.

\section{Potential Applications}

The quantitative model presented in this work is potentially applicable to predicting the entrainment probabilities of sediments in natural channels typically characterized by the near-bed turbulent bursting, grain-size heterogeneity, and random bed configuration. Taking these factors into consideration, the present model can effectively evaluate the entrainment probabilities of mixedsize sediments under various transport conditions. Given the fact that accurate prediction of sediment entrainment is a crucial element of many stochastic bedload models, the entrainment probabilities obtained in this study are most likely to improve the model predictions and extend the applicability to more realistic and complex conditions. For example, in applying a stochastic partial transport model (Wu and Yang 2004) to predict the bedload transport rates of mixed-size sediments, we found that the accuracy obtained with the fourth-order GC pdf is overall $9 \%$ higher than that obtained with the Gaussian pdf.

In addition, the entrainment probabilities of mixed-size sediments are potentially useful for the planning of flushing flows (Wu 2000; Wu and Chou 2003b; Wu and Chou 2004). In release of flushing flows to remove the excess amount of fine sand from the spawning gravel bed, two types of flushing process, i.e., surface and depth flushing, are used. The former removes sand from the bed surface without entraining the gravels, whereas the latter involves the entrainment of surface gravels and thus permits removal of subsurface fine sediments. Depth flushing is usually implemented prior to the spawning season, while surface flushing may be implemented during the incubation period to prevent accumulation of fine sediments. The entrainment probabilities of each size fraction predicted with the present model can be used in the design of various flushing processes. With accurate evaluations of the fractional entrainment probabilities and transport rates associated with different flows, the optimal release scheme could be specified to achieve the maximum flushing efficiency.

\section{Conclusions}

This paper presents a robust methodology for predicting rolling and lifting probabilities of mixed-size sediment under turbulent shear flows. The principal improvements over previous work are the use of a more realistic probability distribution for near-bed streamwise velocity, and a better treatment of mixed grain sizes. The following conclusions are drawn from this study.

1. The near-bed coherent flow structures exhibit consistent correlation patterns characterized by the higher-order moments of velocity fluctuations. For smooth and transitional beds, the second-, third-, and fourth-order moments vary as a function of roughness Reynolds number, whereas these parameters remain constant for fully rough beds.

2. For fully rough beds, the third-order GC pdf can be used to approximate the fourth-order one. However, for smooth beds, use of the fourth-order GC pdf is recommended so that the effects of higher-order correlations are more properly incorporated.

3. For smooth beds the primary difference between the fourthorder GC and normal pdfs is in the kurtosis, while for rough beds the primary difference between the two pdfs is in the skewness. The conventional use of normal pdf for turbulent velocities would lead to an overestimation of kurtosis for the smooth beds but an underestimation of skewness for the rough beds.

4. The skewness and kurtosis of the LN pdf are both greater than those of the corresponding GC pdf, leading to an overestimation in the lower near-mean region but an underestimation in the upper near-mean region. The present model using the fourth-order GC pdf significantly improves the accuracy of lifting probabilities for unisize sediments as com- 
pared to those predicted with the lognormal models, especially at greater dimensionless shear stress.

5. The present model is able to evaluate the entrainment probabilities of mixed-size sediments under partial- and fulltransport conditions, while previous models fail to accurately evaluate the entrainment probabilities for partially transported mixed-size sediments.

In the present study, the instantaneous hydrodynamic forces acting on a sediment particle have been related to the instantaneous streamwise velocity. The instantaneous vertical velocity and the correlation between the two components are not addressed in this work. As we know, turbulent bursting is a twodimensional phenomenon such that use of the streamwise component to characterize the near-bed coherent flow structures is at best a simplified treatment, although the model results are encouraging. In future studies, it is worthwhile to investigate quantitatively the role of instantaneous Reynolds stress on sediment entrainment. To that end, the near-bed two-dimensional coherent flow structures (including the joint pdf and higher-order moments of $u^{\prime}$ and $v^{\prime}$ ) must be fully understood; the mechanistic relations between the Reynolds stress and hydrodynamic forces need to be resolved. With such information, the contributions of four burst events on the entrainment and transport of sediment can be further evaluated.

\section{Acknowledgments}

The work presented herein was supported through the Young Investigator Research Award Grant of National Science Council, Republic of China. Comments from three ASCE reviewers and the Associate Editor helped improve the paper.

\section{Notation}

The following symbols are used in this paper:

$A=$ exposed frontal area;

$B_{L}, B_{R}=$ lifting and rolling thresholds defined by Eqs. (20) and (15);

$C=$ coefficient of linear velocity distribution in Eq. (2);

$C_{D}, C_{L}=$ drag and lift coefficients;

$D=$ uniform grain size;

$D_{i}=$ size of the particle of interest;

$D_{j}=$ size of the upstream adjacent particle;

$D_{k}=$ size of the downstream supporting particle;

$D_{\min }, D_{\max }=$ lower and upper bounds of size distribution;

$D_{n}=$ representative diameter of the $n$th size fraction;

$D_{50}=$ median grain size;

$D_{84}=84$ th percentile grain size;

$\|e\|_{2}=$ Euclidean norm (root-sum-square) of errors;

$F_{D}, F_{L}=$ instantaneous effective drag and lift forces;

$F_{u}=$ flatness (kurtosis) factor of $u^{\prime}$;

$f_{\mathrm{EH}}\left(\Delta_{j}\right)=$ pdf of exposure height;

$f_{\mathrm{FH}}\left(\Delta_{k}\right)=$ pdf of friction height;

$f_{\mathrm{GC} 4}(U)=$ fourth-order Gram-Charlier pdf of $U$;

$f_{s}\left(D_{k}\right)=$ pdf of grain size $D_{k}$;

$f_{u}\left(u_{b}\right)=$ pdf of $u_{b}$;

$k_{s}=$ equivalent roughness of Nikuradse;

$k_{s}^{+}=$roughness Reynolds number $=u_{*} k_{s} / \nu ;$

$L_{D}, L_{L}, L_{W}=$ moment arms of $F_{D}, F_{L}$, and $W$;
$N=$ total number of size fractions;

$P_{L}, P_{R}=$ lifting and rolling probabilities;

$P_{T}=$ total entrainment probability;

$P L, P R=$ mean lifting and rolling probabilities;

$P T=$ mean total entrainment probability;

$P_{e i}, P_{h i}=$ total exposed and hidden probabilities of particles $D_{i}$;

$p_{n}=$ proportion of the $n$th size fraction;

$\mathrm{R}_{p}=$ particle Reynolds number $=\bar{u}_{b} \Delta_{j} / \nu$;

$R^{2}=$ coefficient of determination;

$S_{u}=$ skewness factor of $u^{\prime}$;

$U=$ normalized velocity fluctuation $=u^{\prime} / \sigma_{u}$;

$\bar{u}=$ temporal mean velocity at $y$;

$u_{b}=$ near-bed instantaneous streamwise velocity;

$\bar{u}_{b}=$ area-averaged mean approaching velocity;

$u_{*}=$ bed shear velocity $=\sqrt{\tau_{0} / \rho}$;

$u^{\prime}, v^{\prime}=$ longitudinal and vertical velocity fluctuations;

$W=$ submerged weight of a sediment particle;

$y=$ vertical distance from velocity origin;

$y_{b}=$ location at which $\bar{u}=\bar{u}_{b}$ (=location of effective drag);

$y_{0}=$ virtual zero-velocity level of logarithmic profile in Eq. (3);

$y_{1}, y_{2}=$ lower and upper limits of integration in Eq. (5);

$y^{+}=$normalized height $=u * y / \nu$;

$y_{c}^{+}=$representative coherent height;

$\gamma_{s}, \gamma=$ specific weights of sediment and water;

$\Delta_{j}=$ exposure height;

$\Delta_{k}=$ friction height;

$\delta=$ thickness of roughness layer;

$\theta=$ dimensionless shear stress $=\tau_{0} /\left(\gamma_{s}-\gamma\right) D$;

$\theta_{i}=$ dimensionless effective shear stress based on $D_{i}=\tau_{i} /\left(\gamma_{s}-\gamma\right) D_{i}$;

$\kappa=$ von Karman constant;

$v=$ kinematic viscosity of fluid;

$\xi_{i}=$ hiding factor for particles $D_{i}$;

$\rho=$ density of fluid;

$\sigma_{u}=$ standard deviation of $u^{\prime}$ (=turbulence intensity);

$\tau_{i}=$ effective shear stress applied on particles $D_{i}\left(=\xi_{i} \tau_{0}\right)$; and

$\tau_{0}=$ mean bed shear stress.

\section{References}

Alfredsson, P. H., Johansson, A. V., Haritonidis, J. H., and Eckelmann, H. (1988). "The fluctuating wall-shear stress and the velocity field in the viscous sublayer." Phys. Fluids, 31, 1026-1033.

Andreopoulos, J., and Bradshaw, P. (1981). "Measurements of turbulence structure in the boundary layer on a rough surface." Boundary-Layer Meteorol., 20, 201-213.

Andreopoulos, J., Durst, F., Zaric, Z., and Jovanovic, J. (1984). "Influence of Reynolds number on characteristics of turbulent wall boundary layers." Exp. Fluids, 2, 7-16.

Barndorff-Nielsen, O. (1979). "Models for non-Gaussian variation with applications to turbulence." Proc. R. Soc. London, Ser. A, 368, 501520.

Bridge, J. S., and Bennett, S. J. (1992). "A model for the entrainment and transport of sediment grains of mixed sizes, shapes, and densities." Water Resour. Res., 28(2), 337-363.

Buffington, J. M., Dietrich, W. E., and Kirchner, J. W. (1992). "Friction angle measurements on a naturally formed gravel streambed- 
implications for critical boundary shear-stress." Water Resour. Res., 28(2), 411-425.

Cheng, N.-S., and Chiew, Y.-M. (1998). "Pickup probability for sediment entrainment." J. Hydraul. Eng., 124(2), 232-235.

Cheng, N.-S., and Chiew, Y.-M. (1999). "Closure to 'Pickup probability for sediment entrainment'." J. Hydraul. Eng. 125(7), 789.

Cui, J., Patel, V. C., and Lin, C.-L. (2003). "Large-eddy simulation of turbulent flow in a channel with rib roughness." Int. J. Heat Fluid Flow, 24, 372-388.

Di Cicca, G. M., Iuso, G., Spazzini, P. G., and Onorato, M. (2002). "Particle image velocimetry investigation of a turbulent boundary layer manipulated by spanwise wall oscillations." J. Fluid Mech., 467, 41-56.

Dittrich, A., Nestmann, F., and Ergenzinger, P. (1996). "Ratio of lift and shear forces over rough surfaces." Coherent flow structures in open channels, P. J. Ashworth, S. J. Bennett, J. L. Best, and S. J. McLelland, eds., Wiley, Chichester, 125-146.

Djenidi, L., Elavarasan, R., and Antonia, R. A. (1999). "The turbulent boundary layer over transverse square cavities." J. Fluid Mech., 395, 271-294.

Durst, F., Jovanovic, J., and Kanevce, L. (1987). "Probability density distribution in turbulent wall boundary-layer flows." Turbulent shear flows 5, Springer-Verlag, Berlin, 197-220.

Einstein, H. A. (1950). "The bed load function for sediment transportation in open channel flows." Tech. Bull. 1026, USDA, Washington, D.C.

Fischer, P. F., Leaf, G. K., and Restrepo, J. M. (2002). "Forces on particles in oscillatory boundary layers." J. Fluid Mech., 468, 327-347.

Frenkiel, F. N., and Klebanoff, P. S. (1967). "Higher-order correlations in a turbulent field." Phys. Fluids, 10, 507-520.

Frenkiel, F. N., and Klebanoff, P. S. (1973). "Probability distributions and correlations in a turbulent boundary layer." Phys. Fluids, 16, 725737.

Grass, A. J. (1971). "Structural features of turbulent flow over smooth and rough boundaries." J. Fluid Mech., 50, 233-255.

Grass, A. J., and Mansour-Tehrani, M. (1996). "Generalized scaling of coherent bursting structures in the near-wall region of turbulent flow over smooth and rough boundaries." Coherent flow structures in open channels, P. J. Ashworth, S. J. Bennett, J. L. Best, and S. J. McLelland, eds., Wiley, Chichester, 41-61.

Guy, H. P., Simons, D. B., and Richardson, E. V. (1996). "Summary of alluvial channel data from flume experiments, 1956-1961." USGS Professional Paper, 462-I.

Jain, S. C. (1992). "Note on lag in bedload discharge." J. Hydraul. Eng., 118(6), 904-917.

Johansson, A. V., and Alfredsson, P. H. (1982). "On the structure of turbulent channel flow." J. Fluid Mech., 122, 295-314.

Kim, J., Moin, P., and Moser, R. (1987). "Turbulence statistics in fully developed channel flow at low Reynolds number." J. Fluid Mech., 177, 133-166.

Kirchner, J. W., Dietrich, W. E., Iseya, F., and Ikeda, H. (1990). "The variability of critical shear-stress, friction angle and grain protrusion in water-worked sediments." Sedimentology, 37, 647-672.

Kreplin, H.-P., and Eckelmann, H. (1979). "Behavior of the three fluctuating velocity components in the wall region of a turbulent channel flow." Phys. Fluids, 22, 1233-1239.

Ling, C.-H. (1995). "Criteria for incipient motion of spherical sediment particles." J. Hydraul. Eng., 121(6), 472-478.

Luque, R. F. (1974). Erosion and transport of bed load sediment, Delft University of Technology, Delft, The Netherlands.

Misri, R. L., Garde, R. J., and Ranga Raju, K. G. (1984). "Bed load transport of coarse nonuniform sediments." J. Hydraul. Eng., 110(3), 312-328.

Moin, P., and Kim, J. (1982). "Numerical investigation of turbulent channel flow." J. Fluid Mech., 118, 341-377.

Nakagawa, H., and Nezu, I. (1977). "Prediction of the contributions to the Reynolds stress from bursting events in open-channel flows." J. Fluid Mech., 80, 99-128.
Nelson, J. M., Shreve, R. L., McLean, S. R., and Drake, T. G. (1995). "Role of near-bed turbulence structure in bed load transport and bed form mechanics." Water Resour. Res., 31(8), 2071-2086.

Nezu, I., and Nakagawa, H. (1993). Turbulence in open-channel flows, A. A. Balkema, Rotterdam, The Netherlands.

Nikora, V. I., Goring, D. G., McEwan, I., and Griffiths, G. (2001). "Spatially averaged open-channel flow over rough bed." J. Hydraul. Eng., 127(2), 123-133.

Paintal, A. S. (1971). "A stochastic model of bed-load transport." J. Hydraul. Res., 9(4), 527-554.

Papanicolaou, A. N., Diplas, P., Dancey, C. L., and Balakrishnan, M. (2001). "Surface roughness effects in near-bed turbulence: Implications to sediment entrainment." J. Eng. Mech., 127(3), 211-218.

Papanicolaou, A. N., Diplas, P., Evaggelopoulos, N., and Fotopoulos, S. (2002). "Stochastic incipient motion criterion for spheres under various bed packing conditions." J. Hydraul. Eng., 128(4), 369-380.

Patnaik, P. C., Vittal, N., and Pande, P. K. (1994). "Lift coefficient of a stationary sphere in gradient flow." J. Hydraul. Res., 32(3), 471-480.

Proffitt, G. T., and Sutherland, A. J. (1983). "Transport of non-uniform sediments." J. Hydraul. Res., 21(1), 33-43.

Raupach, M. R. (1981). "Conditional statistics of Reynolds stress in rough-wall and smooth-wall turbulent boundary layers." J. Fluid Mech., 108, 363-382.

Robinson, S. K. (1991). "Coherent motions in the turbulent boundary layer." Аnnu. Rev. Fluid Mech., 23, 601-639.

Rodi, W., Mansour, N. N., and Michelassi, V. (1993). "One-equation near-wall turbulence modeling with the aid of direct simulation data." J. Fluids Eng., 115, 196-205.

Schmeeckle, M. W., and Nelson, J. M. (2003). "Direct numerical simulation of bedload transport using a local, dynamic boundary condition." Sedimentology, 50, 279-301.

Spalart, P. R. (1988). "Direct numerical simulation of a turbulent boundary layer up to $R_{\theta}=1410$." J. Fluid Mech., 187, 61-98.

Sun, Z., and Donahue, J. (2000). "Statistically derived bedload formula for any fraction of nonuniform sediment." J. Hydraul. Eng., 126(2), $105-111$.

van Atta, C. W., and Yeh, T. H. (1970). "Some measurements of multipoint time correlations in grid turbulence." J. Fluid Mech., 41, 169178.

van Rijn, L. C. (1984). "Sediment transport. Part I: bed load transport.” $J$. Hydraul. Eng., 110(10), 1431-1456.

Wilcock, P. R., Barta, A. F., Shea, C. C., Kondolf, G. M., Matthews, W. V. G., and Pitlick, J. (1996). "Observations of flow and sediment entrainment on a large gravel-bed river." Water Resour. Res., 32(9), 2897-2909.

Williams, J. J., Thorne, P. D., and Heathershaw, A. D. (1989). "Measurements of turbulence in the benthic boundary layer over a gravel bed." Sedimentology, 36, 959-971.

Wu, F.-C. (2000). "Modeling embryo survival affected by sediment deposition into salmonid spawning gravels: Application to flushing flow prescriptions." Water Resour. Res., 36(6), 1595-1603.

Wu, F.-C., and Chou, Y.-J. (2003a). "Rolling and lifting probabilities for sediment entrainment." J. Hydraul. Eng., 129(2), 110-119.

Wu, F.-C., and Chou, Y.-J. (2003b). "Simulation of gravel-sand bed response to flushing flows using a two-fraction entrainment approach: Model development and flume experiment." Water Resour. Res., 39(8), 1211.

Wu, F.-C., and Chou, Y.-J. (2004). "Tradeoffs associated with sedimentmaintenance flushing flows: A simulation approach to exploring noninferior options." River Res. Appl., 20(5), 591-604.

Wu, F.-C., and Lin, Y.-C. (2002). "Pickup probability of sediment under log-normal velocity distribution.” J. Hydraul. Eng., 128(4), 438-442.

Wu, W., Wang, S. S. Y., and Jia, Y. (2000). "Nonuniform sediment transport in alluvial rivers." J. Hydraul. Res., 38(6), 427-434.

Wu, F.-C., and Yang, K.-H. (2004). "A stochastic partial transport model for mixed-size sediment: Application to assessment of fractional mobility.” Water Resour. Res., 40(4), W04501. 